ARTICLE

\title{
Development of TES microcalorimeter for high precision spectroscopy of LX-rays from transuranium elements
}

\author{
Makoto MAEDA $^{1 *}$, Takashi YASUMUNE ${ }^{1}$, Hirofumi OGI $^{1}$, Keisuke MAEHATA $^{1}$, Hidehiko ARIMA $^{1}$, \\ Kenji ISHIBASHI ${ }^{1}$, Takahiro UMENO ${ }^{2}$, Keiichi TANAKA ${ }^{3}$, Keisuke NAKAMURA ${ }^{4}$ and Koji TAKASAKI ${ }^{4}$ \\ ${ }^{1}$ Department of Applied Quantum Physics and Nuclear Engineering, Kyushu University \\ 744 Motooka, Nishi-ku, Fukuoka 819-0395 Japan \\ 2 Taiyo Nippon Sanso Corporation, Tsukuba Japan \\ ${ }^{3}$ SII NanoTechnology Inc, Chiba Japan \\ ${ }^{4}$ Japan Atomic Energy Agency, Tokaimura, Japan
}

A TES microcalorimeter was developed for the energy dispersive measurement of X-ray photons of the energy of 10 to $20 \mathrm{keV}$ with the energy resolution better than $100 \mathrm{eV}$ of FWHM value by holding the counting rate greater than 100 counts per second. The fabricated TES microcalorimeter chip was irradiated by LX-ray photons from an ${ }^{241} \mathrm{Am}$ source at a temperature of $150 \mathrm{mK}$. Detection signal pulses with decay time constant of $420 \mu$ s were observed by supplying the bias current of $330 \mu \mathrm{A}$. The energy-resolution of $100 \mathrm{eV} \mathrm{FWHM}$ value was obtained at $17.75 \mathrm{keV}$ by analyzing detection signal pulses.

KEYWORDS: TES microcalorimeter, correction

\section{Introduction}

It is necessary to estimate the internal exposure dose with high precision by monitoring the amount of the intake of plutonium isotopes for the radiation protection of workers in such as spent nuclear fuel reprocessing- and mixed oxide fuel fabrication facilities. Most transuranium (TRU) elements emit LX-ray photons following the $\alpha$ decay. ${ }^{1)}$ Since the energy of LX-ray photons from TRU elements ranges from 10 to 20 $\mathrm{keV}$, the LX-ray photons are expected to be utilized for a TRU monitor. Typical values of energy and emission probability of LX-ray photons are presented in Table $\mathbf{1}^{2}$. The energyresolution better than $100 \mathrm{eV}$ full width at the half maximum (FWHM) value is required to monitor TRU elements by spectroscopic measurements of LX-ray photons. However energy resolution of conventional semiconductor detectors has not been achieved better than $100 \mathrm{eV}$ FWHM value.

A microcalorimeter with superconducting transition edge sensor (TES) has been demonstrated an excellent energy resolution. ${ }^{3) 4}$ In this work, a TES microcalorimeter was developed for the energy dispersive measurement of X-ray photons of the energy of 10 to $20 \mathrm{keV}$ with the energy resolution better than $100 \mathrm{eV} \mathrm{FWHM}$ value by holding the counting rate greater than 100 counts per second.

\section{TES microcalorimeter}

The microcalorimeter is detector which measures the energy of incident X-ray photon by the temperature rise of a device operating at a very low temperature ${ }^{5)}$. Figure 1 shows an operational concept of the microcalorimeter. The microcalorimeter consists of the energy absorber and the thermometer. The energy of the incident X-ray photons is con-

\footnotetext{
${ }^{*}$ Corresponding author, E-mail: MM61@ @une2a.nucl.kyushu-u.ac.jp
}

(C)Atomic Energy Society of Japan
Table 1 Energy and emission probability of LX-ray photons from ${ }^{241} \mathrm{Am}$ and ${ }^{239} \mathrm{Pu}$.

\begin{tabular}{ccccc}
\hline \multirow{2}{*}{ LX-ray } & \multicolumn{2}{c}{${ }^{241} \mathrm{Am}$} & \multicolumn{2}{c}{${ }^{239} \mathrm{Pu}$} \\
\cline { 2 - 5 } & Energy(keV) & $\begin{array}{c}\text { Emission } \\
\text { probability }\end{array}$ & Energy(keV) & $\begin{array}{c}\text { Emission } \\
\text { probability }\end{array}$ \\
\hline $\mathrm{LI}$ & 11.87 & 0.0104 & 11.62 & 0.001163 \\
$\mathrm{~L}_{\alpha 2}$ & 13.76 & 0.017 & 13.44 & 0.00192 \\
$\mathrm{~L}_{\alpha 1}$ & 13.95 & 0.149 & 13.62 & 0.01685 \\
$\mathrm{~L}_{\beta 2}$ & 16.84 & 0.0314 & 16.43 & 0.00354 \\
$\mathrm{~L}_{\beta 1}$ & 17.75 & 0.0837 & 17.22 & 0.01395 \\
$\mathrm{~L}_{\gamma 1}$ & 20.78 & 0.0192 & 20.17 & 0.00316 \\
$\mathrm{~L}_{\gamma 2}$ & 21.10 & 0.0040 & 20.49 & 0.00031 \\
$\mathrm{~L}_{\gamma 3}$ & 21.34 & 0.0039 & 20.72 & 0.000309 \\
$\mathrm{~L}_{\gamma 6}$ & 21.49 & 0.0038 & 20.84 & 0.000612 \\
\hline
\end{tabular}

verted into the temperature rise in the absorber. The raised temperature is decreased to the cold stage temperature by a heat flow through the thermal link. The sensitive thermometer is an important component for achieving excellent energy resolution. The TES microcalorimeter utilizes a strong temperature dependence of the electric resistivity of a superconducting thin film in the phase transition region for a precise measurement of the temperature rise induced by energy deposition of incident X-ray photons.

In this work, the TES microcalorimeter was developed for spectroscopic measurements of LX-ray photons from TRU elements with the energy resolution better than $100 \mathrm{eV}$ of FWHM value by holding the counting rate greater than 100 counts per second. The TES consists of bilayer structure of $\mathrm{Ti}$ and Au thin films. The phase transition temperature of the TES was designed to be $200 \mathrm{mK}$ by the use of the proximity effect between $\mathrm{Au}$ and $\mathrm{Ti}$ with taking the thickness of $\mathrm{Ti}$ and 


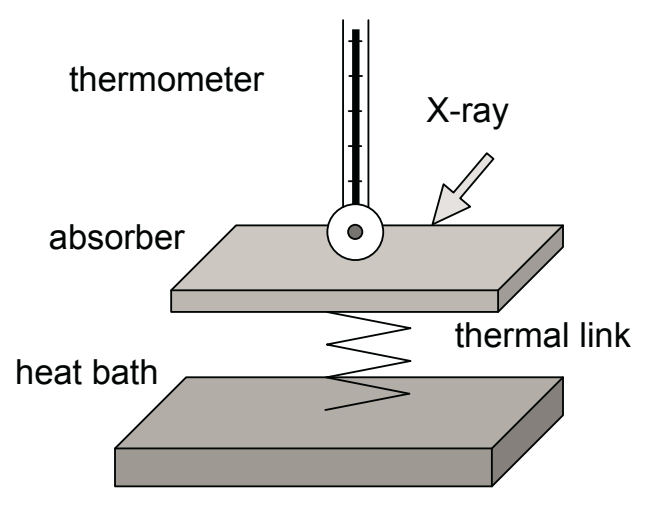

Fig. 1 Model of microcalorimeter.

Au to be 40 and $100 \mathrm{~nm}$ respectively. The Au absorber of 5.0 $\mu \mathrm{m}$ thick is deposited on the Ti/Au bilayer of TES for detecting LX-ray photon of $20 \mathrm{keV}$ with the absorption efficiency of $50 \%$. The geometrical dimensions of the thermometer and the absorber are $300 \times 300 \mu \mathrm{m}^{2}$ and $500 \times 500 \mu \mathrm{m}^{2}$, respectively. TES chip was fabricated by SII NanoTechnology Inc. Figure 2 shows the photograph of fabricated TES chip.

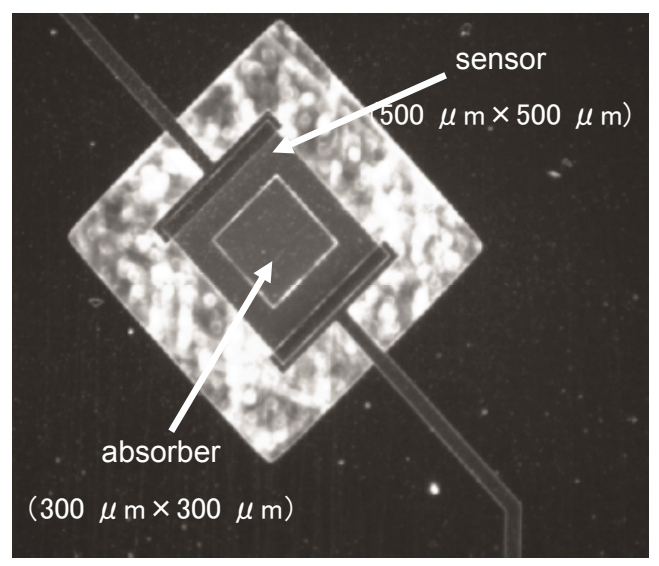

Fig. 2 Photograph of fabricated TES chip.

\section{Optimal filter}

When all detected signal pulses are assumed to have the same shape and consist of an X-ray pulse and a noise components, the detected pulse $D(f)$ in the frequency domain can be expressed as

$$
D(f)=A \times M(f)+N(f)
$$

where $M(f)$ is the normalized model pulse shape, $A$ is an amplitude of the pulse height and $N(f)$ is the noise component ${ }^{6)}$. The minimum value of the variance $\chi$ on $A$ is achieved by minimizing the difference between the detected pulse, $D(f)$, and the model pulse shape $M(f)$. The condition to minimize is

$$
\chi^{2}=\sum \frac{[D(f)-A \times M(f)]^{2}}{N^{2}(f)} .
$$

Setting $d \chi^{2} / d A$ to zero and re-arranging to find the estimated energy of the photon $A$, we obtain

$$
A=\frac{\sum D(f) \frac{M^{*}(f)}{|N(f)|^{2}}}{\sum \frac{|M(f)|^{2}}{|N(f)|^{2}}} .
$$

This expression can be transferred back into the time domain as

$$
A=\frac{1}{C} \sum D(t) T(t)
$$

where $C=\sum|M(f)|^{2} /|N(f)|^{2}$ and $T(t)$ is the optimal filter template. $T(t)$ is constructed by taking the inverse Fourier transform of $M^{*}(f) /|N(f)|^{2}$.

\section{Experimental apparatus}

The TES microcalorimeter was cooled down to the operating temperature by the Liquid-Helium-Free ${ }^{3} \mathrm{He}-{ }^{4} \mathrm{He}$ dilution refrigerator manufactured by Taiyo Nippon Sanso Corporation. Figure 3 shows a schematic drawing of a cross section of the dilution refrigerator.

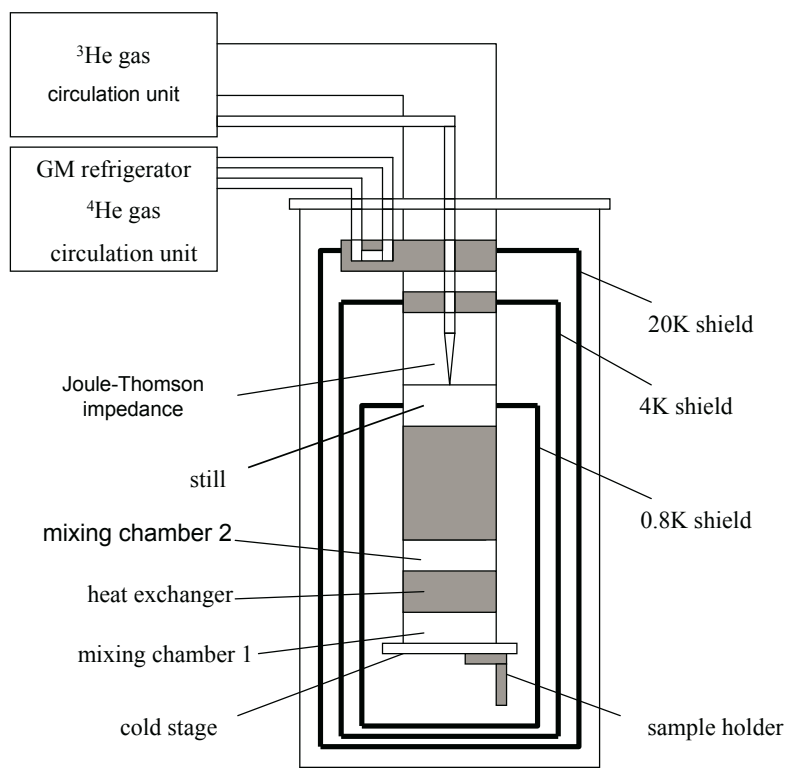

Fig. 3 Schematic drawing of the liquid-helium-free ${ }^{3} \mathrm{He}-{ }^{4} \mathrm{He}$ dilution refrigerator.

Figure 4 shows a circuit diagram of the TES microcalorimeter and the superconducting quantum interference device (SQUID) amplifier. TES is connected to input coil in series and connected to shunt resistor in parallel. TES microcalorimeter was operated with applying a constant DC current. When the temperature rise is caused by energy deposition, the magnetic flux in the input coil decreases with the current flowing through the TES. This change is read out by a SQUID amplifier fabricated by SII NanoTechnology Inc.

\section{V. $R$ - $T$ curve}

The values of output voltage of SQUID was measured with changing temperatures of the cold stage with applying a con- 


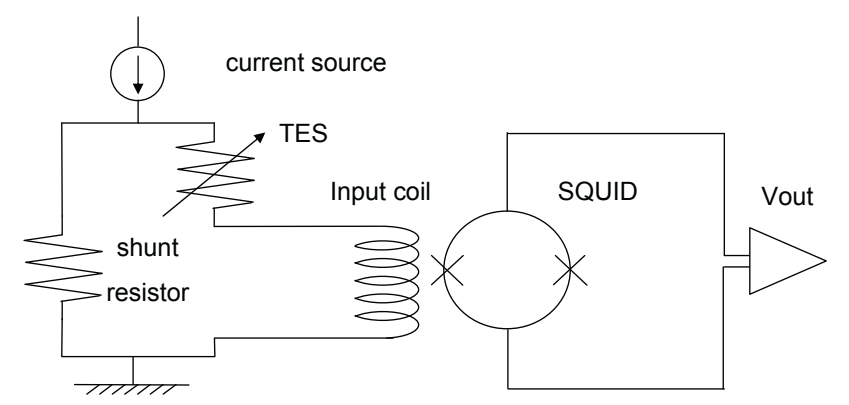

Fig. 4 Circuit diagram of the TES microcalorimeter and the SQUID amplifier.

stant bias current. The measured voltage was converted into the resistance of TES by equation

$$
R_{T E S}=\left(\frac{I_{B}}{\eta V_{\text {out }}}-1\right) R_{\text {shunt }}
$$

where $R_{T E S}$ and $R_{\text {shunt }}$ are electric resistance of the TES and the shunt resistor of $7 \mathrm{~m} \Omega, I_{B}$ is the bias current, $V_{\text {out }}$ is the output voltage of the SQUID amplifier, $\eta$ is a factor of SQUID converting $V_{\text {out }}$ into a current flowing TES.

Constant bias current of 5 and $10 \mu \mathrm{A}$ was supplied to suppress the temperature difference between the TES and heat bath with small Joule heat generation. Figure 5 shows the obtained relationship between the electric resistance of the TES and the temperature ( $R-T$ curve). A phase transition temperature was found to be $208 \mathrm{mK}$ with a width of $12 \mathrm{mK}$.

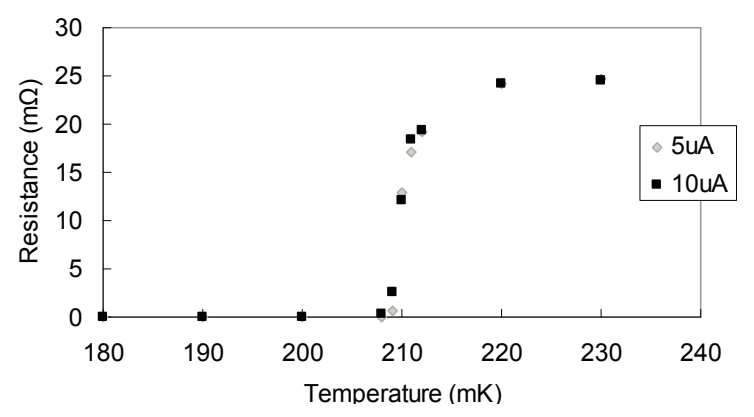

Fig. $5 R$-T curve of the TES microcalorimeter.

\section{VI. $R$-I curve}

The value of the bias current is determined for maintaining the TES in the phase transition region by use of relationship between the electric resistance and the bias current ( $R-I$ curve) at an operating temperature. The voltage output of the SQUID amplifier was measured with changing bias current at a bath temperature of $150 \mathrm{mK}$. The electric resistance of the TES was evaluated by use of eq. (5) as a function of the bias current. Figure 6 shows obtained $R-I$ curve. The TES was found to be maintained in the phase transition region by applying bias current from 300 to $440 \mu \mathrm{A}$. The bias current of the TES was determined to be $330 \mu \mathrm{A}$.

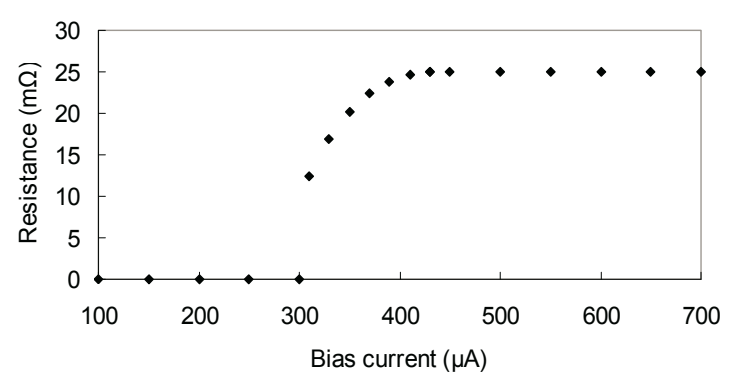

Fig. $6 R$ - $I$ curve of the TES microcalorimeter.

\section{Detection of LX-ray from ${ }^{241}$ Am source}

The fabricated TES microcalorimeter chip was irradiated by LX-ray photons from an ${ }^{241} \mathrm{Am}$ source at a temperature of $150 \mathrm{mK}$. Detection signal pulses with rise and decay time constant of 30 and $420 \mu \mathrm{s}$, respectively were observed by supplying the bias current of $330 \mu \mathrm{A}$. The decay time constant of $420 \mu$ s allows the TES microcalorimeter operating with the counting rate higher than 100 counts per second. Figure 7 shows examples of detection signal pulse. Base line fluctuations were observed in Fig. 7.

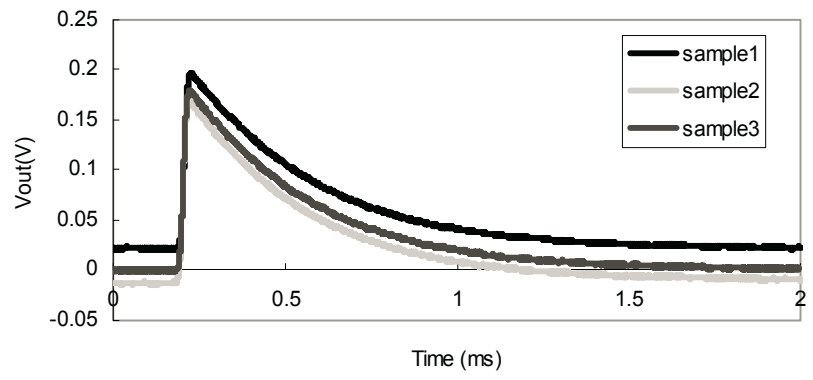

Fig. 7 Voltage signal pulses of the TES microcalorimeter in detection of LX-ray photon from ${ }^{241} \mathrm{Am}$ source.

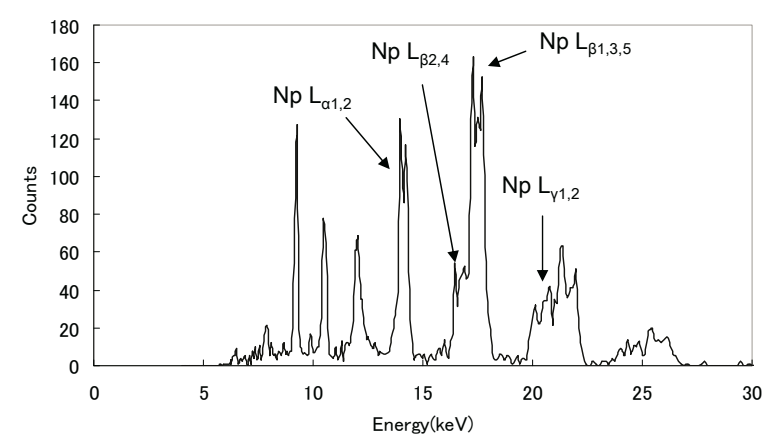

Fig. 8 Pulse height spectrum of LX-ray from ${ }^{241}$ Am source.

Figure 8 shows an energy spectrum of LX-ray photons from the ${ }^{241} \mathrm{Am}$ source obtained from the pulse height distribution of detection signal pulses processed with using a low pass filter with cut-off frequency of $50 \mathrm{kHz}$. The value of the energy-resolution was estimated to be $300 \mathrm{eV} \mathrm{FWHM} \mathrm{value}$ at $\mathrm{L}_{\beta 1} \mathrm{X}$-ray of $17.75 \mathrm{keV}$. 
Figure 9 shows relationship between values of pulse height corresponding to detection of $\mathrm{L}_{\beta 1} \mathrm{X}$-ray photons and the base line level. Pulse height distribution was obtained by calculating eq.(4) with consideration of dependence of the pulse height on the base line fluctuation.

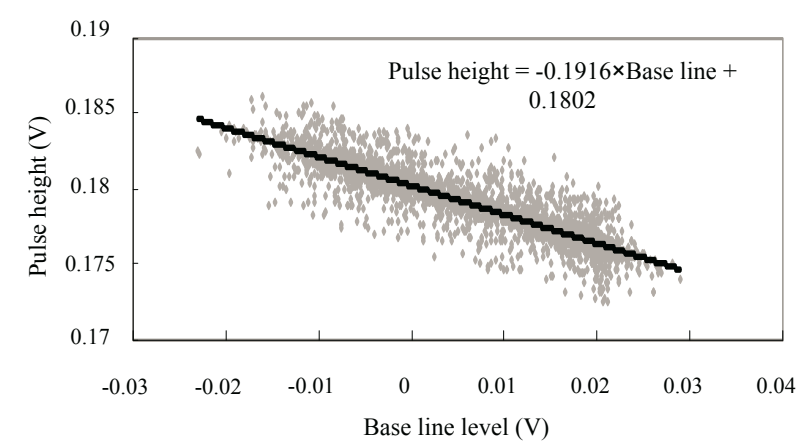

Fig. 9 Relationship between base line and pulse height of detected $\mathrm{L}_{\beta 1} \mathrm{X}$-ray from ${ }^{241}$ Am source.

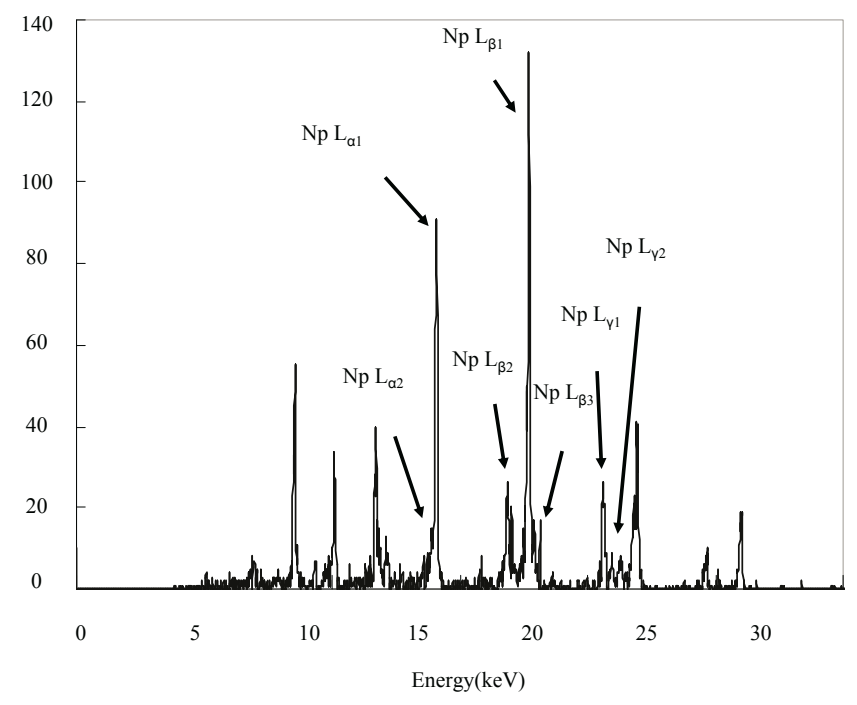

Fig. 10 Energy spectrum of LX-ray from ${ }^{241}$ Am source with base line correction and optimal filter.

Figure 10 shows obtained energy spectrum of LX-ray photons from the ${ }^{241} \mathrm{Am}$ source obtained from the corrected pulse height distribution of detection signal pulses. The value of energy-resolution was improved to be $100 \mathrm{eV}$ FWHM at $\mathrm{L}_{\beta 1}$

\section{$\mathrm{X}$-ray of $17.75 \mathrm{keV}$.}

\section{Conclusion}

The TES microcalorimeter was developed for precise spectroscopic measurement of LX-ray photons from TRU elements. The fabricated TES microcalorimeter chip was irradiated by LX-ray photons from an ${ }^{241} \mathrm{Am}$ source at a temperature of $150 \mathrm{mK}$. Observed detection signal pulses with decay time constant of $420 \mu \mathrm{s}$ allows operating the TES with the counting rate higher than 100 counts per second. The value of the energy-resolution was estimated to be $300 \mathrm{eV} \mathrm{FWHM}$ value at $17.75 \mathrm{keV}$. The energy-resolution was improved to be $100 \mathrm{eV}$ FWHM value by analyzing detection signal pulses with correction of the relationship between base line level and pulse height of signal pulses and using optimal filter.

\section{Acknowledgment}

Authors gratefully acknowledge Y. Nagafuchi, T. Masuda, Y. Ikeda, Y. Ohshima for their support for experiments.

This work is supported in part by Grant-in-Aid for Scientific Research (A) (18206098) of the Japan Society for the Promotion of Science and the JAEA Cooperative Research Scheme on the Nuclear Fuel Cycle.

\section{References}

1) ICRPPu-38,Radionuclide Transformation,Annals of the ICPR,Vol.11 13,Pergamon Press, 1983

2) BNM-LNHB/CEA Table de Radioneucléides, http://www. nucleide.org/DDEP_WG/DDEPdata.htm.

3) J. N. Ullom, J. A. Beall, W. B. Doriese, et al., "11th International Workshop on Low Temperature detectors" Invited Talks, Tokyo, Japan, Jul. 31 and Aug. 1-5 (2005).

4) T. Oshima, Y. Yamakawa, H. Kurabayashi, A. Hoshino, Y. Ishisaki, T. Ohashi, K. Matsuda, K. Tanaka, "A high energy resolution gamma-ray TES microcalorimeter with fast response time" J. Low Temp. Phys., 151 430-435

5) K. D. Irwin, "An application of electrothermal feedback for high resolution cryogenic particle detection" Appl. Phys. Lett., 66[15],1998-2000(1995).

6) A.E. Szymkowiak, R.L. Kelley, S.H. Moseley, C.K. Stahle, J. Low Temp. Phys. 93 (1993) 281. "Signal processing for microcalorimeters" 